\title{
NONCOUNTABLE NORMALLY LOCALLY FINITE DIVISION ALGEBRAS
}

\section{IACOPO BARSOTTI}

A (commutative) field $F$ is regular (see [1] of the bibliography) if it is not finite, and if in addition it is true that the direct (=Kronecker $=$ tensor) product of two normal (=central) division algebras, of finite orders, over $F$ is not a division algebra unless their orders are relatively prime; algebraic number fields and $p$-adic fields are examples of regular fields. A division algebra $\mathfrak{A}$ over a field $F$ is normally locally finite if any finite subset of $\mathfrak{A}$ is contained in a normal (over $F$ ) division sub-algebra of $\mathfrak{A}$ of finite order; in [1], such algebras were called "of type 1." A subisomorphism of an algebra $\mathfrak{A}$ over $F$ is an algebra-isomorphism of $\mathfrak{A}$ into $\mathfrak{A}$, and it is proper if it is not onto. If $\mathfrak{A}$ is a normally locally finite division algebra over the regular field $F$, without a finite basis over $F$, a characteristic sub-algebra of $\mathfrak{A}$ is any normally locally finite division sub-algebra $\mathfrak{D}$ of $\mathfrak{A}$, with countably infinite basis over $F$, having the property that any normally locally finite division sub-algebra of $\mathfrak{A}$, with finite or countable basis over $F$, is isomorphic to a sub-algebra of $\mathfrak{D}$. It was proved in [1] that any $\mathfrak{A}$ of the previous type has a characteristic sub-algebra, unique but for isomorphisms; it was also proved that there exists a normally locally finite division algebra over the regular field $F$, with infinite noncountable basis, and with a given characteristic sub-algebra $\mathfrak{D}$, if and only if $\mathfrak{D}$ admits proper subisomorphisms; [1] contains a rather involved proof of the fact that any $\mathfrak{D}$ admits proper subisomorphisms if $F$ is not countable, and thus establishes the existence of normally locally finite division algebras, with infinite noncountable basis, over any noncountable regular field; this seems to be the only known example of such algebras. We shall present here a very simple proof of the same result, and will, at the same time, dispense with the condition of noncountability of $F$.

(1). Lemma. Let $\mathfrak{A}, \mathfrak{B}, \mathfrak{S}$ be normal division algebras, of finite orders $>1$, over the (certainly infinite) field $F$, and suppose $\mathfrak{A} \times \mathfrak{B} \times \mathbb{S}$ also to be a division algebra; let $m$ be an element of $\mathfrak{A} \times \mathfrak{B}$ but not of $\mathfrak{A}$. Then there exists a $d \in \mathfrak{B} \times \mathfrak{C}$, not zero, such that $d^{-1} m d \in \mathfrak{U} \times \mathfrak{B}$.

In the previous statement, as in the rest of this paper, the identity

Received by the editors April 3, 1957. 
elements of the direct factors of a direct product of algebras are assumed to be coincident.

Proof (being a modification, due to D. Zelinsky, of a proof by the author). Let $c$ be an element of $(5$, but not of $F$, and let $b$ be an element of $\mathfrak{B}$ such that $m b \neq b m$; such $b$ exists because the commutator (= centralizer) of $\mathfrak{B}$ in $\mathfrak{A} \times \mathfrak{B}$ is $\mathfrak{A}$, and $m \in \mathfrak{A}$. Set $x=b c \neq 0$, so that also $1+x \neq 0$; if the lemma is false, we have $x y=m x,(1+x) z=m(1+x)$ for suitable elements $y, z$ of $\mathfrak{A} \times \mathfrak{B}$; subtracting, we obtain $m=z$ $+x(z-y)$. If $y=z$, then $m=z=y, x m=m x, b m=m b$, a contradiction; if $y \neq z$, then $b c=x=(m-z)(z-y)^{-1} \in \mathfrak{H} \times \mathfrak{B}$, also a contradiction, since $c \notin F$, Q.E.D.

For the convenience of the reader, we repeat here a portion of the statement of $(6)$ of $[1]$ :

(2). Lemma. Let $\mathfrak{A}$ be a normally locally finite division algebra, with countably infinite basis, over the field $F$; a necessary and sufficient condition in order that $\mathfrak{A}$ admit a proper subisomorphism is that there exist a factorization

$$
\mathfrak{H}=\mathfrak{B}_{0} \times \mathfrak{B}_{1} \times \cdots
$$

of $\mathfrak{A}$ as a direct product of normal division algebras of finite orders $>1$ over $F$, an $m \in \mathfrak{B}_{0}$, and a sequence $h_{1}, h_{2}, \cdots$ of elements of $\mathfrak{A}$, such that, after setting $\mathfrak{A}_{i}=\mathfrak{B}_{0} \times \mathfrak{B}_{1} \times \cdots \times \mathfrak{B}_{i}$, the following conditions be satisfied:

(a) $h_{i} \in \mathfrak{A}_{i}$;

(b) $h_{i+1}=h_{i} c_{i}$ for a $c_{i} \in \mathfrak{B}_{i} \times \mathfrak{B}_{i+1}$;

(c) there exists no $z_{i-1} \in \mathfrak{A}_{i-1}$ such that $h_{i} z_{i-1}=m h_{i}$.

We can now prove:

(3). Theorem. Let $\mathfrak{A}$ be as in (2); then $\mathfrak{A}$ admits a proper subisomorphism.

Proof. By (29) of $[1],{ }^{1} \mathfrak{H}_{0}$ cannot be transformed into itself by every inner automorphism of $\mathfrak{A}_{1}$; hence there exist an $m \in \mathfrak{A}_{0}$, and an $h_{1} \in \mathfrak{A}_{1}$, with $h_{1} \neq 0$, such that $h_{1}^{-1} m h_{1} \in \mathfrak{A}_{0}$. We shall now proceed to build the sequence $\left\{h_{i}\right\}$ of (2) by induction: assume the $h_{1}, \cdots, h_{i}$ to have been found; by (1) (after replacing $\mathfrak{A}$ by $\mathfrak{A}_{i-1}, \mathfrak{B}$ by $\mathfrak{B}_{i}$, $\mathfrak{S}$ by $\mathfrak{B}_{i+1}, m$ by $\left.h_{i}^{-1} m h_{i}\right)$, there exists a $c_{i} \in \mathfrak{B}_{i} \times \mathfrak{B}_{i+1}$, not zero, such that $c_{i}^{-1}\left(h_{i}^{-1} m h_{i}\right) c_{i} \notin \mathfrak{A}_{i}$. Then $h_{i+1}=h_{i} c_{i}$ satisfies the conditions of (2), Q.E.D.

1 This is the little theorem with a distinguished career, first proved in [2], which later came to be known as the Cartan-Brauer-Hua theorem (see for instance [3, Chapter VII, \$13]); the proof given in [1] is the first elementary proof for the finite case. 
(4). Corollary. Let $\mathfrak{A}$ be as in (2), and assume $F$ to be regular; then there exists a normally locally finite division algebra over $F$, with infinite noncountable basis, having $\mathfrak{A}$ as characteristic sub-algebra.

On the other hand, if $F$ is not regular, the concept of characteristic sub-algebra loses meaning; however, from (3), and from a slight modification of the construction used to prove the sufficiency of (3) of [1], we still obtain:

(5). Corollary. Let $F$ be a field such that there exists a normally locally finite division algebra $\mathfrak{A}$ with countably infinite basis over $F$; then there exists a normally locally finite division algebra over $F$, with infinite noncountable basis, having $\mathfrak{A}$ as a sub-algebra.

Remark. An examination of the proof of (3) of [1] discloses that all the normally locally finite division algebras, with infinite noncountable basis over $F$, whose existence has been established in this note, have a basis of cardinality $\boldsymbol{\aleph}_{1}$; the existence of normally locally finite division algebras over $F$, with a basis of cardinality $>\boldsymbol{N}_{1}$, is still an open problem, at least when $F$ is regular.

\section{BIBLIOGRAPHY}

1. I. Barsotti, Ricerche sopra le algebre divisorie di tipo 1, e sopra le algebre divisorie non algebriche, Rend. di Matem. e sue Appl. vol. 7 (1948) p. 1.

2. H. Cartan, Théorie de Galois pour les corps non commutatifs, Ann. Ecole Norm. vol. 64 (1947) p. 58.

3. N. Jacobson, Structure of rings, Amer. Math. Soc. Colloquium Publications vol. 37, 1956.

University of Pittsburgh 